\title{
COVID-19 and the Risk of Pulmonary Embolism: ECG Findings Can Help
}

Shojaei A and Karimi A*

School of Medicine, Tehran University of Medical

Sciences, Tehran, Iran

*Corresponding author: Amirali Karimi, School of Medicine, Tehran University of Medical Sciences, Tehran, Iran

Received: February 23, 2021; Accepted: March 13, 2021; Published: March 20, 2021

\section{Editorial}

We appreciate the kind invitation to provide an update on an aspect of our study published before [1]. Therefore, we decided to further discuss the Pulmonary Embolism (PE) in patients with COVID-19.

COVID-19 pandemic has affected many countries and their health-care system. COVID-19 also imposes significant diagnostic challenges because of its wide range of complications [2]. Evidence shows that COVID-19 can cause a hypercoagulable state and increases the risk of thromboembolism [3]. The radiological appearance of PE in patients with COVID-19 might differ from COVID-19 (-) patients, and the clots are dominantly seen in peripheral zones $[4,5]$. This fact supports the idea that COVID-19 can cause PE by in-situ immune thrombosis, and the clots might originate from lung vessels rather than deep veins [6]. There is an overlap between signs and symptoms of COVID-19 infection and pulmonary embolism, making the diagnosis of pulmonary embolism challenging [7]. Elevated D-dimer levels may come to our help and increase our suspect for PE, but it is still not specific to the diagnosis of Venous Thromboembolism (VTE) [8]. Anticoagulant therapy may increase the patients' survival rate [9].

Dyspnea, chest pain, and tachypnea are common in both PTE and COVID-19 infection. Hemoptysis has also been described as a rare COVID clinical symptom (0-5\%) [10], while this number is $13 \%$ for patients with PE [11].

In the case reported by Casey et al. [7], a 42 years old COVID-19 positive male presented with pleuritic chest pain, dyspnea, and hemoptysis. In the physical exam, he was tachypneic, and auscultation revealed bibasilar rhonchi. Electrocardiography demonstrated right axis deviation and an S1Q3T3 pattern with flattening of the T-waves in the II, III, aVL and aVF. These findings increased the probability of PE. Also, Laboratory evaluation showed a D-dimer of $4.8 \mu \mathrm{g} / \mathrm{dl}$. So a Computerized Tomography Angiography (CTA) of the chest was obtained. CTA demonstrated bilateral segmental pulmonary emboli and peripheral ground-glass opacities caused by COVID-19 pneumonia.

However, sometimes everything becomes more complicated. In the case reported by Ioan et al. [12], a 61 years old male with a history of hypertension treated with Angiotensin-Converting Enzyme Inhibitor (ACEI) presented with dyspnea and cough. His blood pressure was normal, his heart and respiratory rates were 136 and 30, and oxygen saturation was $<85 \%$ on room air. His Electrocardiogram (ECG) showed sinus tachycardia, ST depression in I, aVL, and V2-V4, and ST-segment elevation in II, III, aVF. An Echocardiography was obtained, which showed RV dilatation, interventricular septal shift, $\mathrm{RV}$ lateral wall akinesia, and a pulmonary arterial systolic pressure of $>60 \mathrm{mmHg}$. His hypoxemia was refractory to invasive mechanical ventilation, so he was intubated and underwent invasive mechanical ventilation without response. For this patient thrombolytic treatment was started due to suspicion of PE. The patient started improving. CTA confirmed PE with a finding of diffuse bilateral ground-glass opacities consistent with COVID-19 pneumonia. A coronary angiogram showed no significant coronary stenosis.

In this case, no obvious sign or symptom, such as pleuritic chest pain or hemoptysis, could lead to PE diagnosis at first. In ECG, there was no flattening of the T-waves or S1Q3T3 pattern, and the patient had a normal axis. Although PE can present with an ST-segment elevation in anteroseptal leads (V1-V4) [13], it is a rare finding that can be mistaken for ST-elevation myocardial infarction. However, in the discussed patient, RV overload and severe pulmonary hypertension with refractory hypoxemia could lead us to PE diagnosis [12].

\section{Conclusion}

In conclusion, diagnosing COVID-19 complications such as PE can be challenging as COVID-19 can mask their sign and symptoms. We should be aware of any signs or symptoms that can lead us to diagnose these complications. ECG is a low-cost and widely available tool that may give valuable clues to help us diagnose PE. Some ECG signs raise suspicions for $\mathrm{PE}$, such as right axis deviation, S1Q3T3 pattern, or the flattening of $\mathrm{T}$ waves. As we saw in an above-discussed case, even an ST-segment elevation in anteroseptal leads can lead us to $\mathrm{PE}$ diagnosis.

\section{References}

1. Mehraeen E, Seyed Alinaghi SA, Nowroozi A, Dadras O, Alilou S, Shobeiri P, et al. A systematic review of ECG findings in patients with COVID-19. Indian Heart J. 2020; 72: 500-507.

2. Driggin E, Madhavan MV, Bikdeli B, Chuich T, Laracy J, Biondi-Zoccai G, et al. Cardiovascular Considerations for Patients, Health Care Workers, and Health Systems During the COVID-19 Pandemic. J Am Coll Cardiol. 2020; 75: 2352-2371.

3. Han H, Yang L, Liu R, Liu F, Wu KL, Li J, et al. Prominent changes in blood coagulation of patients with SARS-CoV-2 infection. Clin Chem Lab Med. 2020; 58: 1116-1120.

4. Ooi MWX, Rajai A, Patel R, Gerova N, Godhamgaonkar V, Liong SY. Pulmonary thromboembolic disease in COVID-19 patients on CT pulmonary angiography - Prevalence, pattern of disease and relationship to D-dimer. Eur J Radiol. 2020; 132: 109336.

5. van Dam LF, Kroft LJM, van der Wal LI, Cannegieter SC, Eikenboom J, de Jonge $\mathrm{E}$, et al. Clinical and computed tomography characteristics of COVID-19 associated acute pulmonary embolism: A different phenotype of thrombotic disease? Thromb Res. 2020; 193: 86-89. 
6. Cuker A, Tseng EK, Nieuwlaat R, Angchaisuksiri P, Blair C, Dane K, et al. American Society of Hematology 2021 guidelines on the use of anticoagulation for thromboprophylaxis in patients with COVID-19. Blood Advances. 2021; 5 : 872-888.

7. Casey K, Iteen A, Nicolini R, Auten J. COVID-19 pneumonia with hemoptysis: Acute segmental pulmonary emboli associated with novel coronavirus infection. Am J Emerg Med. 2020; 38: 1544.e1-1544.e3.

8. Zhou F, Yu T, Du R, Fan G, Liu Y, Liu Z, et al. Clinical course and risk factors for mortality of adult inpatients with COVID-19 in Wuhan, China: a retrospective cohort study. Lancet. 2020; 395: 1054-1062.

9. Tang N, Bai H, Chen X, Gong J, Li D, Sun Z. Anticoagulant treatment is associated with decreased mortality in severe coronavirus disease 2019 patients with coagulopathy. J Thromb Haemost. 2020; 18: 1094-1099.

10. Huang C, Wang Y, Li X, Ren L, Zhao J, Hu Y, et al. Clinical features of patients infected with 2019 novel coronavirus in Wuhan, China. Lancet. 2020 395: 497-506.
11. Stein PD, Terrin ML, Hales CA, Palevsky HI, Saltzman HA, Thompson BT, et al. Clinical, laboratory, roentgenographic, and electrocardiographic findings in patients with acute pulmonary embolism and no pre-existing cardiac or pulmonary disease. Chest. 1991; 100: 598-603.

12. Ioan AM, Durante-López A, Martínez-Milla J, Pérez-Calvo C, Santos A Pulmonary embolism in COVID-19. When nothing is what it seems. Rev Esp Cardiol (Engl Ed). 2020; 73: 665-667.

13. Falterman TJ, Martinez JA, Daberkow D, Weiss LD. Pulmonary embolism with ST segment elevation in leads V1 to V4: case report and review of the literature regarding electrocardiographic changes in acute pulmonary embolism. J Emerg Med. 2001; 21: 255-261. 